\title{
Formation and Evolution of Galaxies: Starlight Synthesis Algorithm
}

\author{
Nick Barua \\ Swift Xi Inc., Kobe City, Japan \\ Email: nick.barua@swift-xi.com
}

How to cite this paper: Barua, N. (2022) Formation and Evolution of Galaxies: Starlight Synthesis Algorithm. International Journal of Astronomy and Astrophysics, 12, 68-93. https://doi.org/10.4236/ijaa.2022.121005

Received: December 23, 2021

Accepted: February 28, 2022

Published: March 3, 2022

Copyright $\odot 2022$ by author(s) and Scientific Research Publishing Inc. This work is licensed under the Creative Commons Attribution International License (CC BY 4.0).

http://creativecommons.org/licenses/by/4.0/

\begin{abstract}
This study will see the resurgence of interest in precise velocity dispersion measurements, both for the study of galactic and active nuclei kinematics. As several works suggest, an excellent tactic to measure $\sigma$ is to use the absorption lines of the calcium triplet, as it is a spectral region relatively free from complications. The discovery of an empirical relationship between the mass of the

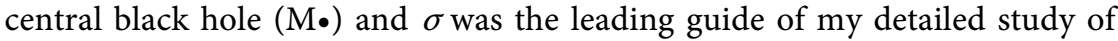
the calcium triplet region. This search for more accurate methods to calculate the dispersion of velocities, in addition to the careful study of uncertainties. After investing so much time in the development and improvement of the method and its application to so many galaxies, it is time to reap the rewards of this effort, using my results to address a series of questions concerning the physics of galaxies.
\end{abstract}

\section{Keywords}

Galaxies, Physics, Nuclei Kinematics, Galactic Nuclei, Active Nuclei, Velocity Dispersion

\section{Introduction}

A significant fraction of the galaxies in the universe is distributed in groups and clusters, making these large structures ideal environments for studying the evolution of their member galaxies. The transition between groups and clusters is quite subtle, given by the number of galaxies present in each system. Although clusters are richer, clusters are more abundant in the universe. While only $10 \%$ of the matter in the universe is found in clusters, around $50 \%$ is found in clusters [1]. Groups have about $5-100$ bright galaxies within a radius of $1 \mathrm{~h}-1 \mathrm{Mpc}$ and scattering speeds around $500 \mathrm{~km} / \mathrm{s}$. Already clusters can have hundreds of galaxies distributed in a radius of 1 to $2 \mathrm{~h}-1 \mathrm{Mpc}$ and with dispersion speeds as high 
as $\sim 1000 \mathrm{~km} / \mathrm{s}$ [2]. The typical mass of groups is on the order of $1013 \mathrm{M}$ [3].

Groups and clusters of galaxies are considered to be the largest gravitationally bound structures in the universe. The formation of these objects is based on the $\Lambda \mathrm{CDM}$ model in which small fluctuations of primordial density would have grown over time, giving rise to smaller objects that, in turn, evolved through mergers and accretions to form the most prominent structures known today. However, the virialization of these systems occurs from energy exchanges that occur until the system's dynamic balance is reached. This section will describe the most relevant time scales on which physical phenomena occur in these structures [4]. This study will see the resurgence of interest in precise velocity dispersion measurements, both for the study of galactic and active nuclei kinematics. As several works suggest, a good tactic to measure $\sigma$ is to use the absorption lines of the calcium triplet, as it is a spectral region relatively free from complications [5].

\section{Kinematics of Active Cores of Galaxies}

Experimental evidence allows associating the image of a swarm of bees to the movement of stars in a galaxy under the action of a po ,co of central potential. This random movement is reflected in the galactic spectrum, whose spectral lines of absorption, produced in stellar atmospheres, appear enlarged by the Doppler effect [6]. The broadening of the spectral lines basically depends on the distribution of stellar velocities, according to the equation:

$$
I(\lambda)=\int\left(\lambda^{\prime}=1 / 1=v / c\right) f(v) \mathrm{d} v
$$

where $\lambda$ is the wavelength, $I(\lambda)$ is the observed galactic spectrum, $F\left(\lambda_{0}\right)$ is the stellar spectrum, $c$ is the speed of light, and $f(v)$ is the func distribution of speeds [6]. Assuming a Gaussian distribution, with a dispersion of stellar velocities $\sigma$, the Doppler broadening can be quantified by $\Delta \lambda \sim \lambda_{0} \sigma / c$, where $\lambda_{0}$ is the central wavelength of the line. In this way, if we can measure the Doppler broadening of the spectral lines of a galaxy, we can infer their dispersion of stellar velocities. In Figure 1, we compare the spectrum of a star and that of a galaxy in the calcium triplet region; note how the lines of the galaxy are much wider than those of the star [7].

In a dynamically relaxed system, the virial theorem guarantees that the potential energy $\mathrm{V}$ of the system is directly linked to the kinetic energy $\mathrm{K}$, so that $\mathrm{V}=$ $-2 \mathrm{~K}$. Therefore, stellar velocity dispersion, as it is a measure of kinetic energy, is directly linked to the mass of the system, which is a measure of potential energy. In the central regions of a galaxy $(\mathrm{r} \sim 102-3 \mathrm{pc})$, this mass essentially represents the mass in stars M. If we have a measure of the luminosity and size of the system, we can calculate the mass/luminosity ratio, M/L. The M/L ratio, in turn, can be used to diagnose stellar populations. Just remember that for main-sequence stars, $\mathrm{L} \sim \mathrm{M} 4$; this implies that younger populations have a lower M/L ratio than older populations [7].

It is not just the mass/lightness ratio. However, that can be inferred from 


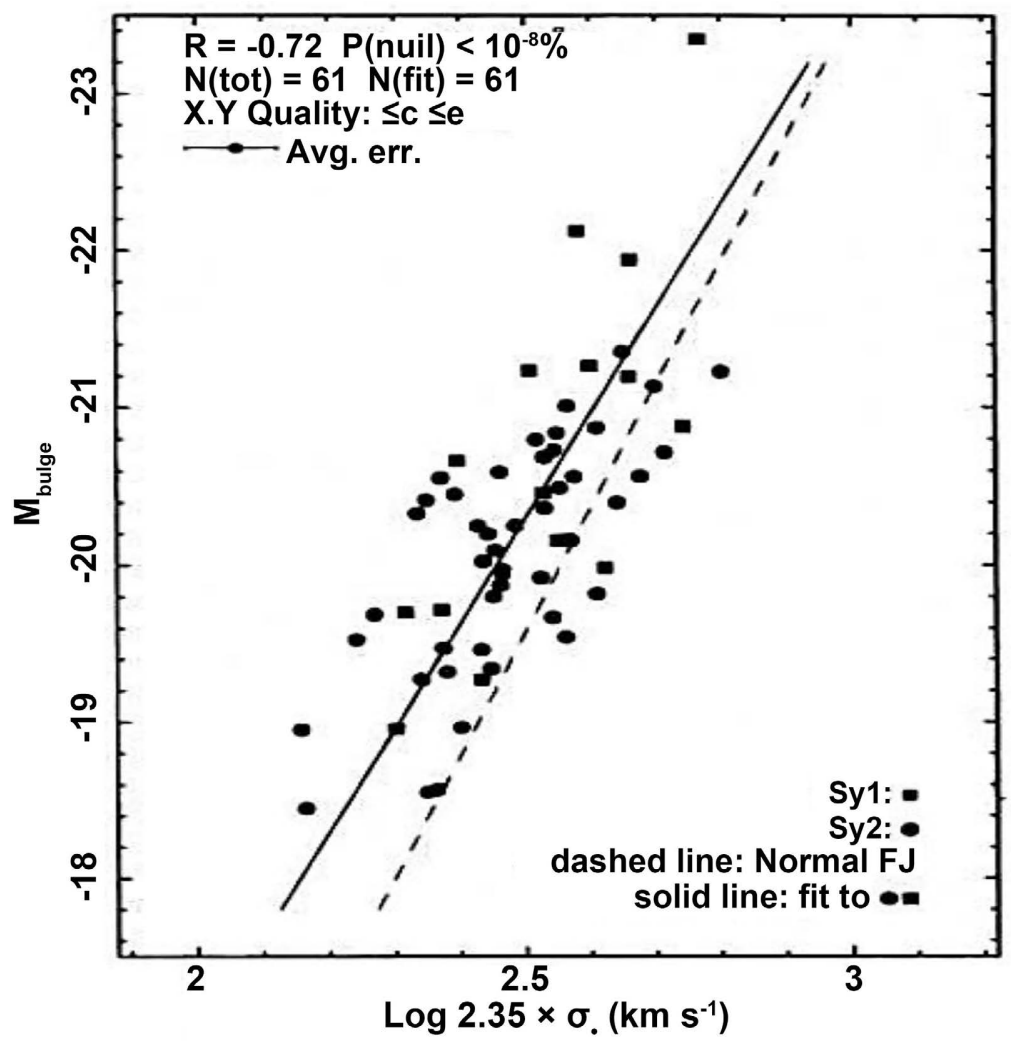

Figure 1. Faber-Jackson relation for Seyfert-type galaxies. On the abscissa axis, we have the magnitude of the bulge in band B (Mbulge); in the ordinate, log $2.35 \times \sigma\left(\mathrm{km} \cdot \mathrm{s}^{-1}\right)$. The solid line represents the best fit to the data; the dotted line, the Faber-Jackson relation for normal galaxies derived by Whittle (1992). Extracted from Nelson \& Whittle 1996ApJ... 465...96N Page 96 (harvard.edu).

galactic kinematics and velocity dispersion [8]. The studies by Kirby et al. revealed a good empirical correlation between $\sigma$ and the mass M• of the supermassive black hole, present at the center of most galaxies with a spheroidal component (i.e., ellipticals or early-type spirals) [9]. Although $\sigma$ as a good central potential tracer, it is not completely intuitive to discover a relationship between black holes in active nuclei of galaxies (AGNs) and the kinematic properties of the gal. Host Axia, since the mass of the central black hole, is much smaller than the mass of stars in the bulge. It is speculated that this relationship, in fact, indicates a connection between the processes of formation and/or evolution of black holes and their host galaxies. Therefore, the kinematics of a galaxy, measured mainly through $\sigma$, carries very useful information about the central potential well, the stellar populations, and even about the activity of AGNs. In the next section, we will review the most relevant studies done to date on stellar kinematics in AGNs [10].

\section{Previous Work}

The Work by Terlevich et al. pioneered the systematic study of the absorption lines of the calcium triplet 8498.02, 8542.09, 8662.14 $\AA$ in AGNs. This spectral 
region stands out for being relatively clean, free from strong emission lines and other spurious effects. These authors emphasized the equivalent width of the calcium triplet (WCaT) and showed that it is a great tool for the diagnosis of stellar populations and the presence of a continuum non-stellar (featureless continuum) [11]. They came to the conclusion that WCaT for Seyfert-type galaxies does not suffer dilution and that, therefore, the featureless continuum disappears from the optical to the near-infrared. This is interpreted as a sign that the $\mathrm{Ca}$ ii lines are produced by red supergiants in bursts of star formation (starbursts). This result was one of the first indications that nuclear activity and stellar formation can coexist in AGNs, a theme that, at the time, caused great controversy due to the clash between defenders of the starburst model for AGN and advocates of the more orthodox (and nowadays hegemonic) school that links the energy source in AGNs to black holes [12].

Another way to study stellar populations is via the mass/luminosity ratio. Oliva et al. estimated M/L for AGN host galaxies by analyzing the $\mathrm{CO}(1.62,2.29$ $\mu \mathrm{m})$ and $\mathrm{Si}(1.59 \mu \mathrm{m})$ bands in the near-infrared. It was observed, then, that the $\mathrm{M} / \mathrm{L}$ ratio in this spectral range is an excellent diagnostic to distinguish between the presence of red supergiant (evidence of recent starbursts) and that of red giants (old population) [13]. Another result of this study is that about $40 \%$ of Seyfert 2 have features of young populations, in agreement with studies in other spectral ranges, such as Cid Fernandes et al. [14]. Interestingly, none of the 8 Seyfert 1 studied by Oliva et al. [15] present evidence of recent stellar formation, contradicting the expectation that Seyferts 1 and 2 differ only in orientation. Unfortunately, to date, this study has not been repeated for a significant sample of objects [16].

It was from the Work of Nelson and Whittle, however, that the dispersion of velocities in the calcium triplet region gained notoriety. In this study, velocity dispersions were measured for 85 objects with the cross-correlation method, for the absorption lines of the calcium triplet and Mg b $\lambda \lambda 5167.5,5172.7,5183.6 \AA$ [17]. The main contribution of this Work was the investigation of the relationships between 1) the stellar kinematics of the bulge and the properties of the active core galaxies, and 2) the stellar kinematics and the gaseous kinematics in these galaxies.

1) The relationship between the stellar kinematics of the bulge and the properties of the active-core host galaxies was explored through the Faber-Jackson relation, $\mathrm{L} \propto \sigma \mathrm{n}$, for Seyfert-type galaxies. For normal galaxies, it was known that $\mathrm{n} \sim 3-4$; for Seyfert galaxies, these authors found $\mathrm{n} \sim 2.7$. That is, there is no significant difference between the Faber-Jackson relation for normal galaxies and for Seyfert galaxies and, therefore, the bulge of Seyfert galaxies is kinematically normal. The authors [18] [19] [20] [21] found, however, an offset in the Faber-Jackson relation for Seyfert galaxies Figure 2 so that the velocity dispersions are on average $20 \%$ smaller than for normal galaxies given the same luminosity. That is, given the same brightness, the Seyfert galaxies are less massive; or, given the same mass, the Seyfert galaxies are more luminous. This implies 


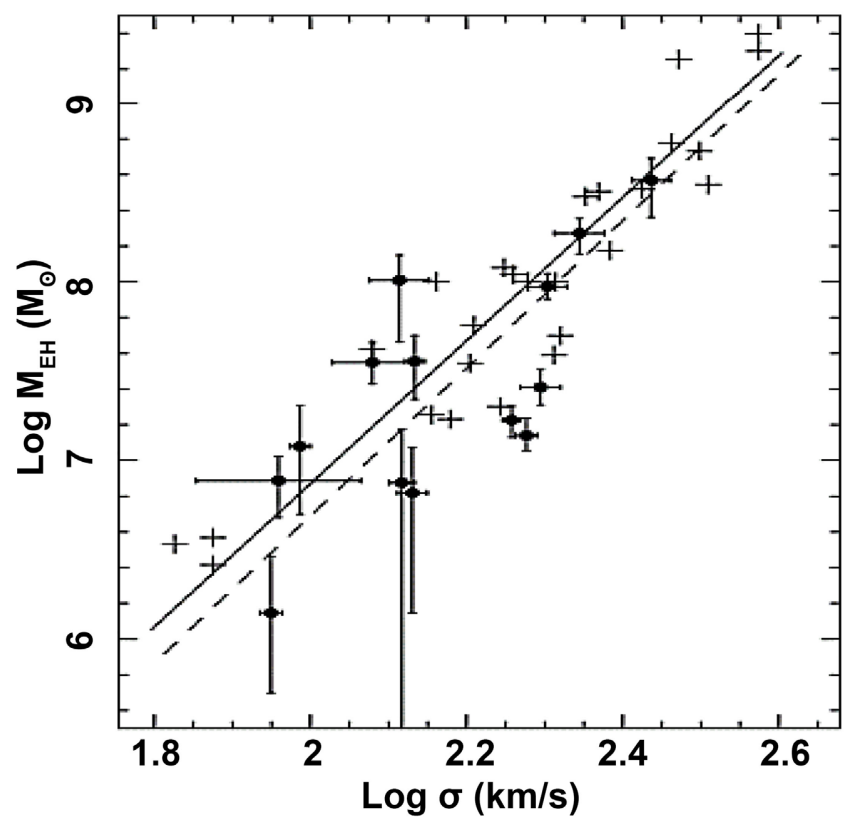

Figure 2. Blackhole mass as a function of velocity dispersion. The circles represent measurements made by Nelson et al. (2004) for Seyfert-type galaxies; the crosses represent measurements by Gebhardt et al. (2000) for normal elliptical and S0 galaxies. Nelson et al. (2004) determined M• through reverberation maps; Gebhardt et al. (2000) obtained them from models of stellar kinematics in the core. The solid line represents the relation $\sigma-\mathrm{M}$ • calculated by Tremaine et al. (2002); the dashed line is the best fit to the chart data. Extracted from Nelson et al. (2004). The black hole mass-stellar velocity dispersion correlation: bulges versus pseudo-bulges (http://www.silverchair.com).

that the M/L ratio for Seyfert galaxies is smaller than for normal galaxies. This result is consistent with starbursts and a young stellar population.

2) Another important result of this Work is the relationship between stellar and gaseous kinematics [22]. A strong correlation was found between the width ogas of the emission lines of [O iii] $\lambda 5007$ and the dispersion of stellar velocities $\sigma$. This implies that the narrow-line region (NLR) has a strong dependence on the central gravitational potential. From these conclusions, we started to use ogas as an alternative to measuring $\sigma$. In order to investigate in which situations can ogas replace $\sigma$, Greene \& Ho (2005) calibrated the ogas- $\sigma$ For various transitions ([O ii] $\lambda 3727,[\mathrm{O}$ iii] $\lambda 5007$ and [S ii] $\lambda \lambda 6716,6731$ ), and for 1749 objects from the sdss database. The Work concluded that $\sigma$ gas could be used as a $\sigma$ plotter; however, because of the scattering of the relation, it should only be used in statistical studies and not for individual objects [22]. They also evaluated the effect of the fiber opening used in the sdss observations in $\sigma$. For the studied sample, the result is that the rotation effect on $\sigma$ is despicable. These were the works of Ferrarese and Merritt [10] and Gebhardt et al. [12], however, which revived the interest in velocity dispersions. Both studies showed that there is a good correlation between this and the mass of the central supermassive black hole [13]. As this relationship is the most direct and probably the only relatively reliable way to measure $\mathrm{M} \bullet$ for distant galaxies, it is not surprising the resurgence of works 
aiming to obtain measurements most accurate for $\sigma$ in AGNs. In the next section, it presents the contribution to this search.

Another additional advantage of working with this spectral range is the diagnostics on stellar populations that can, in principle, be derived from the equivalent width of the Ca ii triplet. Therefore, the importance of deriving reliable methods for measuring velocity dispersion is indisputable. In studies initiated by Vega and Garcia-Rissmann et al., we present an atlas of calcium triplet spectra for 78 objects, most of which are active-core host galaxies [23].

We measured nuclear stellar velocity dispersions for 72 objects with the crosscorrelation and direct fit methods, and we verified that there is no significant difference between the results of the two methods [24]. Measurements were also taken of the equivalent width of the calcium triplet. In the present Work, details of the methods used to measure velocity dispersions were published in Garcia-Rissmann et al. The particularities of each method adopted are presented here, in addition to the extensive tests involved in uncertainty calculations [25]. The main objective is to promote a deeper understanding of the merits and limits of the methods used. The present level of detail will contribute to this deepening by clarifying and adding some points from the studies already published. It should be noted that, for the direct fit method, we use the starlight stellar population synthesis code. Although this is not a synthesis application and we are only interested in $\sigma$, our code proved to be more than adequate for this purpose. For a sub-sample of 34 of our objects, I also present an unprecedented study with spatially resolved spectra. We observe, with these data, the variation of $\sigma$ and of the equivalent width of Ca ii with the spatial position. From the behavior of these parameters, we obtained some diagnoses about stellar populations in Seyfert 1 and 2 galaxies [26].

\section{Sloan Digital Sky Survey}

The Great Databases Heaven has always enchanted the human mind with its tireless cycle of days and nights. Nights, in particular, have always been conducive to speculation about the seemingly endless bright spots. Since the admiration and cataloging of stars made by our most remote ancestors, humanity has sought, with the help of these vast data, to find cohesion and patterns that would explain the phenomena of the universe. Furthermore, when treading these paths, he came across a universe so immense that he had no choice but to subjugate himself and continue cataloging [27]. The efforts to catalog the celestial objects were not small; they came from the Babylonians, passed through Ptolemy, and expanded with photographic plates from the beginning of the 20th century. With the increasing storage capacity and the new possibilities of the so-called digital age, grandiose catalogs are being produced. The Two Degree Field Galaxy Redshift Survey (2dfgrs) and the Sloan Digital Sky Survey (sdss) are some of the boldest projects that have emerged in recent times [28]. Large databases with good quality data allow much more robust statistical tests, essential for corroboration, refutation, or even theory creation. Large databases are also revolutionizing the 
way astronomers work. What used to be done in an almost artisanal way-certain types of reduction and data analysis, for example-nowadays needs innovative and automated approaches [29]. New computational and statistical solutions are increasingly in vogue. sdss, in particular, intends to map an area of $\pi$ stereo-radians (i.e., a quarter of the sky) and catalog more than 100 million objects. Spectral information will be obtained for about a million galaxies in the local neighborhood selected among these objects [30]. The spectra cover the region of 3800 - $9200 \AA$, with resolution $\lambda / \Delta \lambda \sim 1800$, and are observed through 3 -arcsecond aperture fibers. More details about sdss objectives and procedures are found in York et al. (2000). Data reduction and sequencing algorithms are described in Stoughton et al. (2002), data releases, and Adelman-McCarthy et al. The next section provides a small review of studies catalyzed by sdss.

\section{Previous Work}

The abundance of sdss objects is reflected in the immense amount of studies carried out with this database. It is an almost superhuman task to review all studies derived from this base, despite their relative youth - the first wave of data was released in 2001. We will therefore focus on the Work of three groups: 1) Kauffmann et al. [23], 2) Heavens et al. [27]; Panter et al. [28], and 3) Cid Fernandes et al. [29]; Matthew et al. [30]; Stasi'nska et al. [31].

These groups work with the main stellar population synthesis methods applied to sdss objects to recover the history of stellar formation and galactic properties. The studies by Kauffmann et al. [23] retrieve the history of stellar formation, stellar masses, and dust extinction of sdss objects from spectral indices such as the break at $4000 \AA$ and the Balmer's $\mathrm{H} \delta$ absorption line, quantified by the in dices $\operatorname{Dn}(4000)$ and $\mathrm{H} \delta \mathrm{A}$, respectively [29]. In a nutshell, the method of this group consists of comparing the measured indices with a library of star formation histories. The first step of the analysis is to find the spectrum that best models the stellar contribution to the galactic spectrum. Thus, one can separate the galactic spectrum into two: a purely stellar spectrum, in which stellar indices are measured, and a "purely" nebular spectrum, resulting from the subtraction between the observed spectrum and the stellar modeled, in which nebular emission lines are measured [30]. After calculating the spectral indices, we then use the indices $\mathrm{Dn}(4000)$ and $\mathrm{H} \delta \mathrm{A}$ as diagnostics for starbursts and to determine the stellar age; and, from the broadband photometry, the extinction by dust and the mass in stars are recovered [31]. These parameters are obtained by comparing the measurements of indices and colors with a library of Monte Carlo realizations of different histories of star formation, which take into account, various metallicities, both continuous star formation and starbursts. These authors present some important results regarding the mass distribution in galaxies and the host galaxies of AGNs.

The authors [32] found that most of the mass of the local universe resides in galaxies of M? $\sim 5 \times 1010 \mathrm{M}$. Furthermore, there is a clear division between high 
and low mass galaxies Figure 3. Low-mass galaxies generally have a young population, a typical disk concentration index, recent starbursts, and evidence that the rate of star formation is larger than the galactic halo, perhaps as a result of supernova feedback processes. High-mass galaxies have older populations, a typical bulge concentration index, evidence that the rate of star formation decreases the larger the halo, and an indication that there is little star formation oncemassive galaxies form. For the studies of narrow-line AGNs (i.e., Seyferts 2 and LINERs), this group showed that their hosts have properties very similar to those of early-type galaxies [32].

For low luminosity AGNs (calculated from the emission line [O iii] 25007 ), the stellar populations are very similar to those of early-type galaxies; on the other hand, for high luminosity AGNs (L[O iii] > $107 \mathrm{~L}$ ), populations appear to be much younger, and there is evidence of recent starbursts. Based on the study of broad-line AGNs, these authors claim that there is no difference [32] between the stellar composition of galaxies of the Seyfert 2 type and quasars with the same luminosity and redshift. This means that young stellar populations are characteristic of AGNs with high luminosity. Previous studies, such as the one by Cid Fernandes et al., already pointed to this conclusion, but only with the extraordinary statistics of sdss can it be confirmed beyond any doubt. Multiple Optimized Parameter Estimation and Data compression (moped) is another stellar population synthesis method that has been applied to the sdss dataset. According to Heavens et al. and Panter et al., the technique consists of modeling the entire spectrum of a galaxy to retrieve a certain set of parameters about the history of stellar formation, the evolution of metallicity, and extinction.

The first step in the analysis of the moped is the removal of the emission lines and the degradation of the spectra to $\Delta \lambda \sim 20 \AA$, that is, the same resolution as the spectra of simple stellar populations used in the base. The compression algorithm then reduces the spectra to just $\sim 25$ data points which, in principle, contain as much information as possible about the parameters of interest. These 25 points are chosen as the dot product between $\sim 25$ different weight vectors and the vector constructed from the spectral data. The secret is, therefore, in finding weight vectors in order to privilege the wavelengths most sensitive to the parameters of interest (i.e., age, metallicity, and extinction) [32]. According to the authors, this process manages to conserve all the information on the spectrum, or at least all the information of interest; therefore, certain degeneracies, such as age-metallicity, are much milder than for studies with spectral indices. Furthermore, it is worth remembering that no a priori hypothesis is made about the history of star formation.

The authors of studies with the moped obtain some results with implications for the evolution of galaxies. One of the main results is that more massive galaxies form stars sooner compared to less massive galaxies, and therefore the history of star formation is not the same for galaxies of different masses Figure 4 . This points to downsizing behavior. They also find that the rate of star formation has been declining for about 6 billion years; for redshifts of order $z>2$, the 


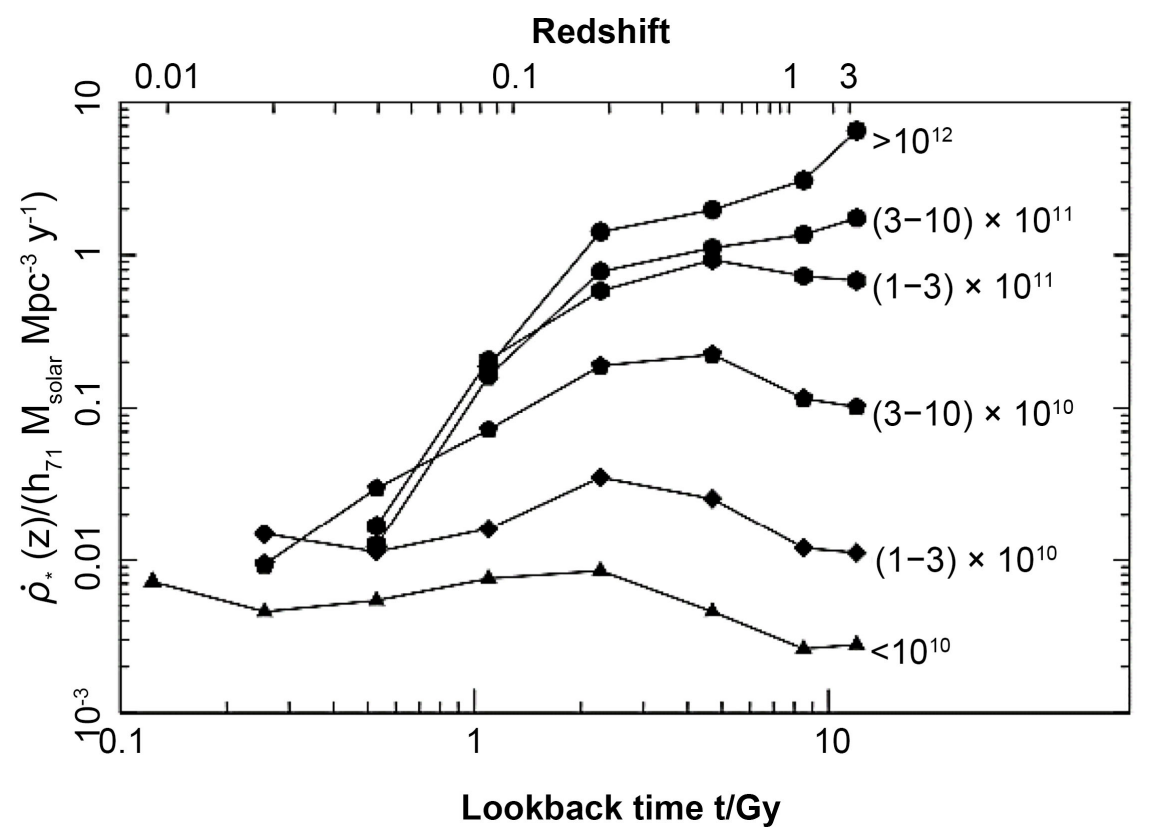

Figure 3. Rate of star shape at different times as a function of star mass in galaxies. Extracted from Heavens et al. [27].
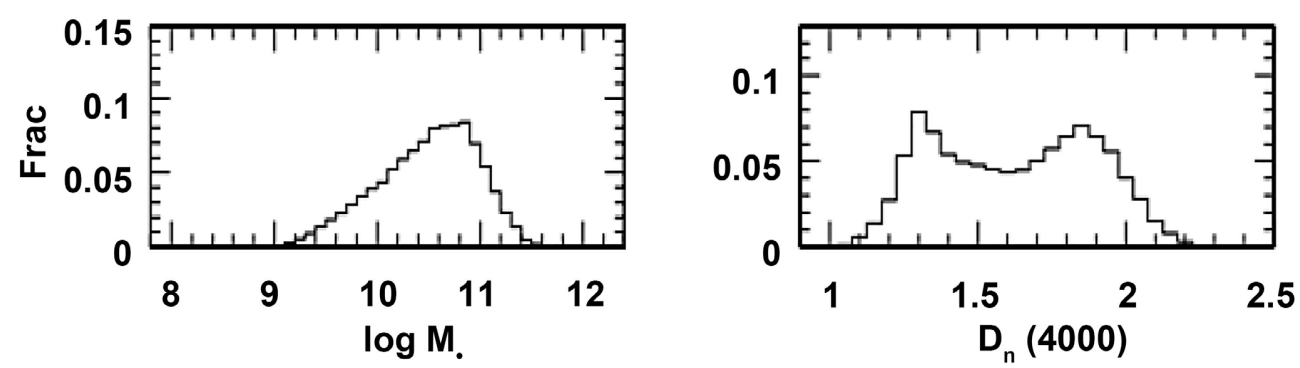

Figure 4. The fraction of total stellar mass in galaxies in the local universe as a function of stellar mass (left panel) and $\mathrm{Dn}(4000)$ (right panel). Dn(4000) is used as a diagnosis for stellar populations, so the higher $\mathrm{Dn}(4000)$, the younger the populations. Note how this distribution is bimodal-extracted from Kauffmann et al. [23].

rate of star formation calculated by the authors is in agreement with observations and independent studies on high redshifts, which is expected by the Copernican principle. Furthermore, the authors state that the distribution of metallicities for stellar-forming gas is inconsistent with closed-box models but consistent with the infall model, by the galaxy. They then calculate the fraction of the mass of the gas in relation to the total mass from infall models. The studies by Cid Fernandes et al. [29]; Matthew et al. [30]; Stasi'nska et al. [31]; Gomes [32] presented some results obtained with the semi-empirical synthesis of stellar populations, using the starlight code, for samples of about 20,000 - 50,000 galaxies of sdss. Spectral synthesis models the entire galactic spectrum as a linear combination of elements from a base of simple stellar populations of different ages and metallicities. Spectra with resolution $\Delta \lambda \sim$ two $\AA$ are used, and the calculated parameters are condensed a posteriori in order to obtain a more robust descrip- 
tion of the galactic properties. The mass and light fractions of each base component, the total mass in stars, stellar kinematics parameters, and the extinction by dust are recovered.

From the residual spectrum, calculated by subtracting the observed and the synthetic spectrum, information is obtained about nebular kinematics, such as the intensity of emission lines, nebular abundances and classification of objects into normal galaxies with stellar formation (NSFG), active cores (AGN), hybrid and passive. Cid Fernandes et al. verified the reliability of this synthesis method to retrieve galactic properties. Simulations with fictitious galaxies, empirical tests for the verification of astrophysical correlations, comparisons of measurements obtained from methods were performed. Independent, in addition to comparison with results from other groups. It was shown in this Work that the retrieved parameters are quite satisfactory, as long as the descriptions are made from a robust combination of parameters and not using the super-detailed results of the synthesis. Gomes [32] carried out an extensive study on the problem of synthesis for elliptical galaxies. It was concluded that these galaxies must have a population with a different $\alpha / \mathrm{Fe}$ ratio from the solar one and that it was not well represented by the stellar population base. They were simply used in the synthesis. This shows that we must be very careful with our results, always checking that they are not being distorted by some effect of incompletion in the base.

Matthew et al. [30] highlight the study of the bimodal distribution of stellar populations in galaxies. This bimodality is seen mainly in the color of the galaxies, in the $\operatorname{Dn}(4000)$ index, and in the middle star age. It is found that the average age weighted by luminosity is the main responsible for this effect. Furthermore, evidence of downsizing is also shown. Stasi'nska et al. [29] used the nebular results to study ways to distinguish AGNs from NSFGs. The emphasis of this Work is to understand why diagnostic diagrams take the form they do, comparing data with photoionization models. The diagram [O ' iii]/ $\mathrm{H} \beta$ versus $[\mathrm{N}$ ii] $/ \mathrm{H} a$ is mainly used. A new dividing line to separate AGNs from NSFGs is proposed. In addition, a new diagram, allows the classification of galaxies at high redshifts with optical spectra. There are, therefore, considerable differences between the methods applied by our group, such as starlight, and the techniques of Kauffmann et al. [23] and Heavens et al. [27]. The group involved in the study by Kauffmann et al. [23], on the one hand, uses spectral indices to create libraries of stellar formation histories, which limits the level of detail in studies of the evolution of galaxies. On the other hand, as they obtain a pure nebular spectrum by subtracting a stellar continuum, they can investigate in-depth the emission lines coming from the gas in galaxies (and from there, for example, study nebular abundances and classify galaxies in AGNs and NSFGs).

The group working with the moped [27], on the other hand, obtains much more robust information about the contribution of stellar populations to galaxies. Therefore, they get a reasonably detailed history of star formation and galactic evolution. However, as their technique consists of completely eliminating the 
emission lines, the entire nebular study carried out by this group is based on chemical evolution models and, therefore, does not reach the degree of detail achieved by Kauffmann et al. (2003a). Starlight, in turn, brings together the best of both worlds and with a greater degree of detail. Our studies of the history of star formation in galaxies, for example, are based on a much more detailed adjustment of the galactic spectrum than the one used by the moped. And, in the study of emission lines, we have at our disposal, for comparison, much more robust stellar parameters than those obtained only with spectral indices, as the Work of Kauffmann et al. We, therefore, have much more complete tools for dealing with various astrophysical problems. In the next section, we present some extensions of the application of the starlight synthesis code to the sdss database, continuing the studies by Cid Fernandes et al. [29]; Matthew et al. [27]; Stasi'nska et al. [30]; Gomes [29].

\section{Current Work}

In this Work, we apply the starlight synthesis algorithm to 354,992 galaxies from the sdss database [27] [32] [33]. We present here two studies made from this sample of galaxies. The first study is dedicated to the technical improvement of the synthesis. Our main aim was to solve the problem of automatic mask creation (that is, the elimination of unwanted regions in the fit) for the spectra of the database objects. Good masks are fundamental to improve the reliability of the fit and obtain an excellent residual spectrum, in which we can more clearly observe nebular emission lines. The second part of this Work represents the beginning of exploring the extensive database built with the synthesis results for 354,992 galaxies. Such a database makes it possible to address numerous astrophysical questions about the nature and evolution of galaxies. To illustrate this potential, we present two preliminary results. First, we use diagnostic diagrams to investigate the differences between the sequences of host galaxies of active cores and normal galaxies with stellar formation. In the second, we obtain the history of the stellar formation of galaxies as a function of their mass in stars for both AGNs and NSFGs. Although many details have not been considered at this time, we already see some fascinating results, which we intend to deepen in further studies.

\section{Synthesis of Stellar Populations}

A hundred years ago, there was still no clear distinction between the Milky Way and the rest of the universe. It was only in the 1920s, mainly from the studies of Edwin Hubble, that it was accepted that some of the "spiral nebulae" observed in the sky were, in fact, other galaxies. The fact that we have somehow discovered galaxies so recently speaks volumes about the difficulty of observing them. Of course, our understanding of extragalactic objects has advanced enormously in the last century, going through the Hubble diagram and into large-scale structure studies. Still, one difficulty remains: for most galaxies, except for the very 
close ones, we cannot resolve individual stars. They still seem, even to the eyes of the most modern telescopes, interesting and curious foggy [26].

Despite the observational difficulty, astronomers have developed some tricks to obtain information about the composition of galaxies, even without being able to solve their constituents. If we can have information about the ions that make up a star even without observing the ions, why shouldn't we be able to deduce which stars make up a galaxy even without being able to directly observe its stars? The strategy found was to reproduce or model the integrated spectrum of a galaxy and, in this process, find the population of stars that inhabit it. Thus, two schools emerged that developed the so-called synthesis of stellar populations: the evolutionary and the semi-empirical.

A detailed review of these studies is made by Gomes [32]. In general terms, evolutionary stellar population synthesis produces a library of spectra from certain initial hypotheses, such as the history of stellar formation, the evolution of which imic, and the initial mass function. An attempt is then made to find, within this library, the spectrum that most resembles the observed galactic spectrum. Semi-empirical stellar population synthesis, on the other hand, attempts to model the spectrum of a galaxy as a linear combination of stars or stellar clusters. Whichever method is adopted, the vast majority of studies do not work with the entire spectrum, only with spectral indices (like equivalent widths and colors, for example). Obviously, both approaches have their advantages and disadvantages.

The main drawback of evolutionary synthesis is to ensure that initial hypotheses lead to real physical models and that the object library is complete. The difficulty of the semi-empirical synthesis, on the other hand, is to start with a base of stars or clusters comprehensive enough to reproduce the different conditions of the other galaxies. And regardless of the approach to the synthesis problem, it is convenient to use the observed spectrum as a whole, which certainly contains more information than isolated spectral indices. Our group decided to tackle the synthesis problem in order to make the best of both schools. We thus try to model the entire spectrum of a galaxy from a linear combination of base elements. This is, at first glance, just a step forward for the semi-empirical synthesis, which has only recently been done with spectral indices. We can, however, insert a dash of evolutionary synthesis if we wish.

The base need not necessarily consist of observed stars; simple theoretical stars or stellar populations can be used. All the hypotheses of chemical evolution, atmospheric models, and stellar formation history will be embedded in this theoretical basis. Despite the great myriad of applications already covered with our synthesis program from observed starbases, only from the library of simple stellar populations developed by Bruzual \& Charlot [34] is that the relevant galactic properties, such as age, metallicity, and mass, could be retrieved in a more robust way. This, added to the large databases with quality spectra, such as the Sloan Digital Sky Survey (sdss), encouraged the improvement of the starlight 
synthesis algorithm [29].

In this work, we apply the starlight synthesis code to two studies. One of these is the study of stellar kinematics in galaxies in the calcium triplet region, at $8498.02,8542.09,8662.14 \AA$. The other is a demonstration of a small fraction of the information that can be derived from the sdss database. In the next section, we will generally discuss the mathematical implementation of our synthesis code. We analyze your idiosyncrasies and care that should be taken when analyzing the results inferred by our algorithm.

\section{The Starlight Synthesis Algorithm}

As explained in the previous section, the mathematical approach of the starlight population synthesis code follows, in a way, the semi-empirical school. Our method consists of modeling an observed $O_{\lambda}$ spectrum using a convex combination (i.e., a linear combination with positive coefficients) of the elements of a base. We've also included a Gaussian to account for kinematics and a term for extinction by dust, according to the following equation:

$$
M_{\lambda}=M_{\lambda 0}\left[\sum_{j=1}^{N_{*}} x_{j} T_{j} r_{\lambda}\right] \otimes G\left(v_{*}, \sigma_{*}\right)
$$

where: $\bullet M_{\lambda}$ is the synthetic spectrum. $\bullet M_{\lambda 0}$ is a normalization factor, defined as the total flux of the synthetic spectrum at wavelength $\lambda_{0} \cdot \bullet T_{j, \lambda}$ is the spectrum of the $j$ th $(j=1, \cdots, N)$ base component, normalized to $\lambda_{0}$. The basis can be constituted either by observed stars or combinations of stars or by theoretical stars or stellar populations. One can even include, depending on the problem studied, other components in the base, such as quasars or power laws. $\bullet X_{j}$ is the fraction that each element $T_{j, \lambda}$ of the base contributes to the flow of $M_{\lambda}$.

- $r_{\lambda} \equiv 10-0.4\left(A_{\lambda}-A_{\lambda 0}\right)$ takes into account the effects of extinction by dust.

- $G(v, \sigma)$ is a Gaussian distribution of line-of-sight velocities, centered on $V$ and enlarged by $\sigma$.

- $\otimes$ expresses a convolution.

The best fit is defined as the one that minimizes the $\chi$ two between the observed spectrum and the model:

$$
\chi^{2}=\sum_{\lambda}\left[\left(O_{\lambda}-M_{\lambda}\right) w_{\lambda}\right]^{2}
$$

where $W_{\lambda}$ is the inverse of the noise in $O_{\lambda}$. We use the Metropolis algorithm in conjunction with simulated annealing (see, for example, MacKay 2003) to try to prevent the fit from being locked by local minimums of $\chi^{2}$. Spectral features that are not desirable in the adjustment, either because they are too noisy or because they are not included in the base, can be masked with the simple definition of $w_{\lambda}$ $=0$.

As a general rule, we verify that masks based on general characteristics of the studied sample produce a good first approximation for the modeled spectra. However, for a refinement of the adjustments, it is essential to make an individual mask for each studied object, taking into account the peculiarities of each spectrum to be modeled. The fact that "general" masks, that is, applied to all ob- 
jects in a sample, produce good results in a first approximation lies in the control mechanisms designed in the program. One of these mechanisms, for example, consists of automatically masking parts of the spectrum that are from two to three sigma (this number is adjustable by the user) above the noise. Thus, it is expected to reduce the interference in the adjustment by stronger emission lines or bad pixels that were eventually not masked a priori by the user. Simulations and empirical tests to verify the reliability of this method in the recovery of galactic properties were performed by Gomes [32]. In an analysis of the results of any application of the synthesis code, one must take into account, for example, the effect of the multiplicity of solutions. This is mainly linked to intrinsic base degenerations (such as the age-metallicity effect or the use of very similar stars) or even statistical degenerations, that is, the impossibility of differentiating two solutions distinct due to the noise level in the studied spectrum.

\section{Applications of the Stellar Population}

Although the starlight synthesis code was developed mainly with the objective of recovering the star formation history of a galaxy, he proved to be much more versatile. The algorithm can be used, for example, to remove all the stellar contributions to a spectrum in order to study the pure nebular emission spectrum of a galaxy. Another possibility is to use it to model only a small spectral band that contains absorption lines and obtain good measurements for the dispersion of stellar velocities of this line. The study presents the application of starlight to measuring the dispersion of stellar velocities in the calcium triplet range. We use standard starbases of observed velocities in the same instrumental configuration as our objects. The bases are mainly constituted by stars of the spectral type $\mathrm{K}$, more frequently of the type KOIII, and eventually some stars of the type G and F.

In these cases, we clearly have the problem of the intrinsic linear dependence of the base. We analyzed the extent to which this affects our measures of velocity dispersion and the corresponding uncertainties. In this study, we used an extensive and extremely detailed theoretical base of 150 simple stellar populations from the Work of Bruzual \& Charlot [34] of 25 different ages and six metallicities. Depending on the degree of precision desired, we use either reduced bases, which save a lot of computation time for the hundreds of thousands of sdss objects, or more complete bases, including a finer grid of populations. Different ages and metallicities. This will actually be the first application of this giant sample, whose modeling was recently completed. Figure 5 shows an example of the application of our synthesis code to the study of the calcium triplet. The following Figure 6 and Figure 7 provide examples of spectral synthesis for sdss database objects.

\section{Study of the Calcium Triplet}

This chapter presents a study of the stellar dynamics of galaxies by analyzing the absorption lines of the calcium triplet 8498.02, 8542.09, 8662.14 ̊. Section 

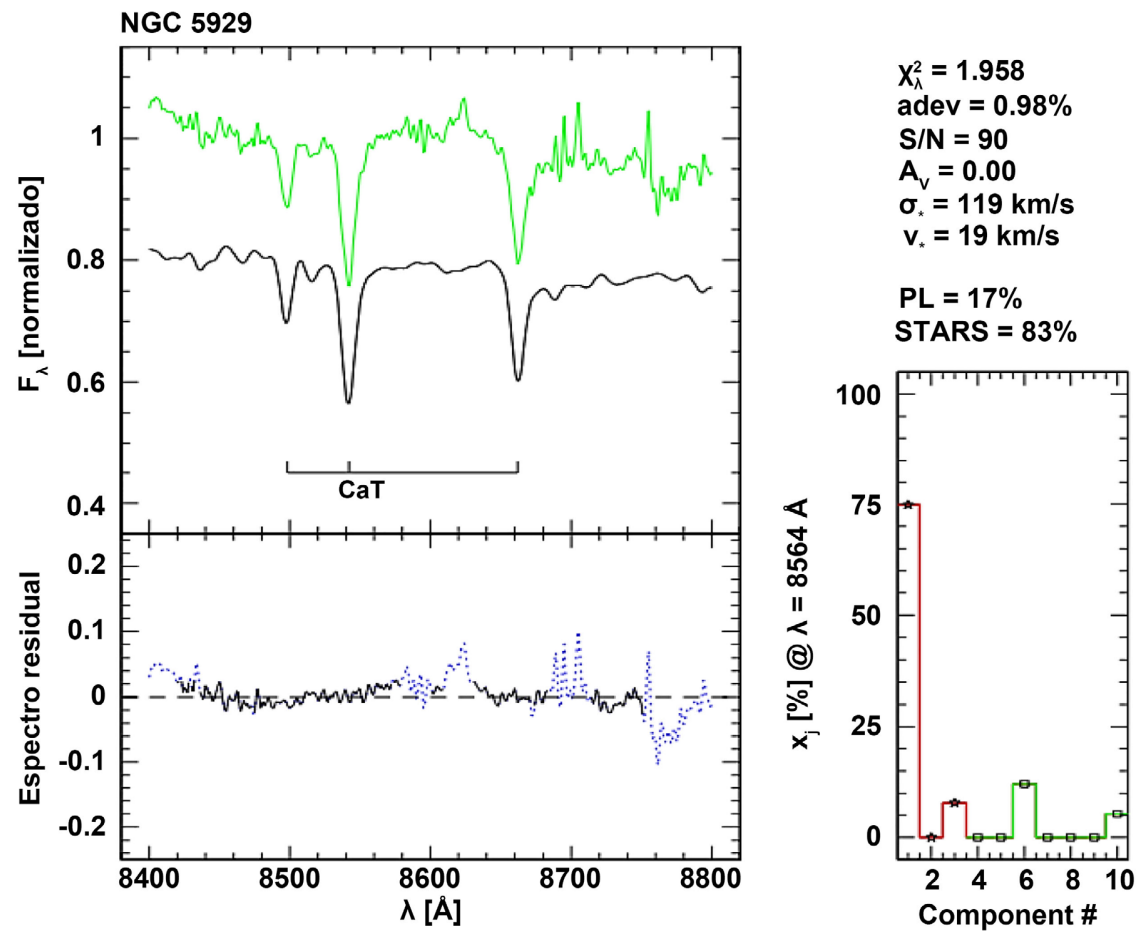

Figure 5. Application of the starlight synthesis code to the study of the calcium triplet. In the upper left panel, we show the observed spectrum of the NGC 5929 galaxy (in green), and the modeled spectrum (in black) shifted down by 0.2 for greater clarity of the figure. The wavelengths of the calcium triplet are marked with the symbol "CaT". The lower panel shows the residual spectrum $O_{\lambda}-M_{\lambda}$. The parts drawn in the dotted line were masked and are not considered in the fit. The right panel shows the contribution of each of the base elements (in this case, standard velocity stars and power laws) to the modeled spectrum. Above this panel are some galactic properties derived by the synthesis program. Among them, we are especially interested in the dispersion of stellar velocities $\sigma$.

3.1 deals with sampling, observations, and data reduction. Despite having followed this process, this part of the Work was carried out almost entirely by collaborators [13]. This last section also detailed a work conducted by me and supervised by Dr. R. Cid Fernandes, taking advantage of the algorithm for calculating the equivalent width of the Ca ii triplet encoded by Vega [20].

\section{Observations and Data Reduction}

The observations present in this Work were made in six shifts in three different telescopes. The following were observed:

- 16 galaxies, in two shifts, by Dr. R. Gonz'alez-Delgado at the KPNO observatory, with the Mayall $4 \mathrm{~m}$ telescope and the Ritchey-Chr'etien spectrograph (Lapi et al., 2018);

- 25 galaxies, in two shifts, by Dr. H. Schmitt at the KPNO observatory, with the $2.1 \mathrm{~m}$ telescope and the Goldcam spectrograph;

- 39 galaxies, in two shifts, by Dr. R. Cid Fernandes and by L. R. Vega [20] at the ESO-La Silla observatory, with the $1.52 \mathrm{~m}$ telescope and the Boller \& Chivens spectrograph. 

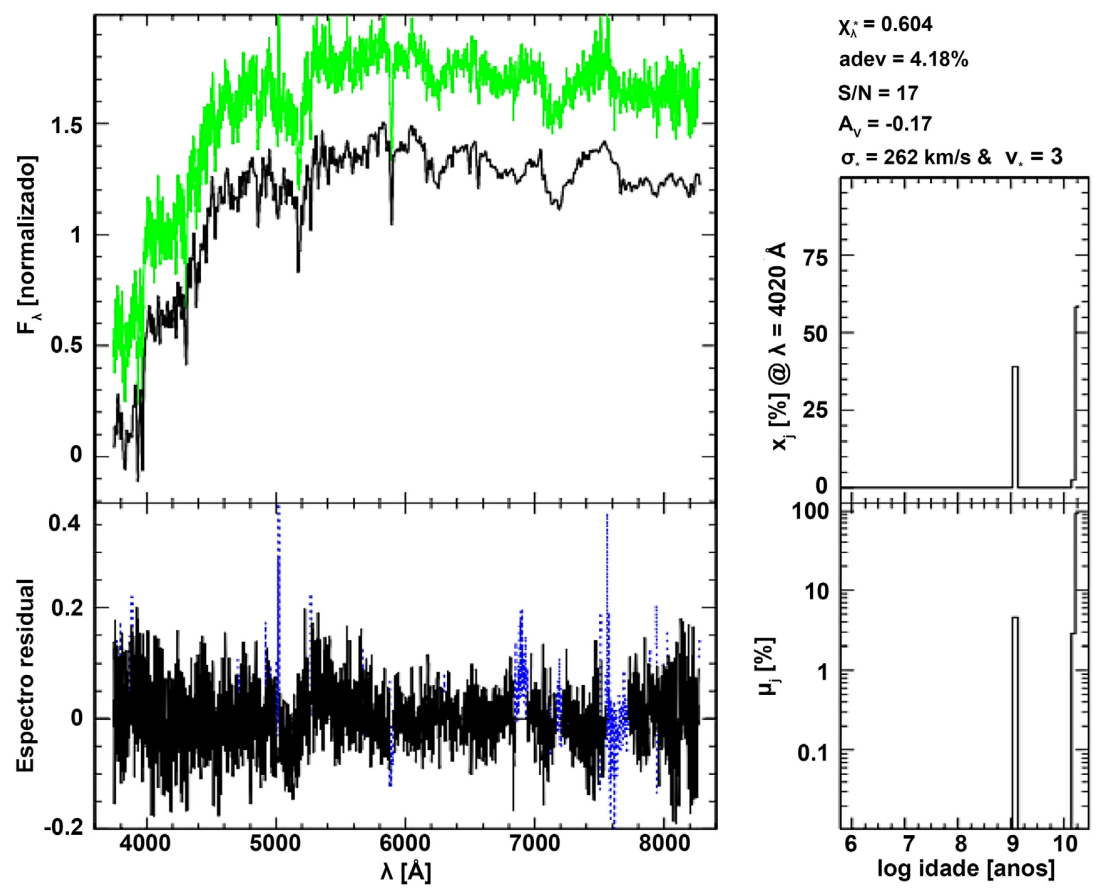

Figure 6. Application of the starlight synthesis code to an early-type sdss database galaxy. In the upper left panel, we show the observed spectrum of the galaxy SDSS J131844.94 011108.20 (in green), and the modeled spectrum (in black) shifted down by 0.4 for greater clarity of the figure. The lower panel has the residual $O_{\lambda}-M_{\lambda}$ spectrum. The parts drawn in the dotted line were masked and are not considered in the fit. The panels to the right show the flux and mass fractions as a function of stellar age. Above these panels are some galactic properties derived by the synthesis program. Semi-empirical analysis of Sloan Digital Sky Survey galaxies-I. Spectral synthesis method (http://www.silverchair.com).
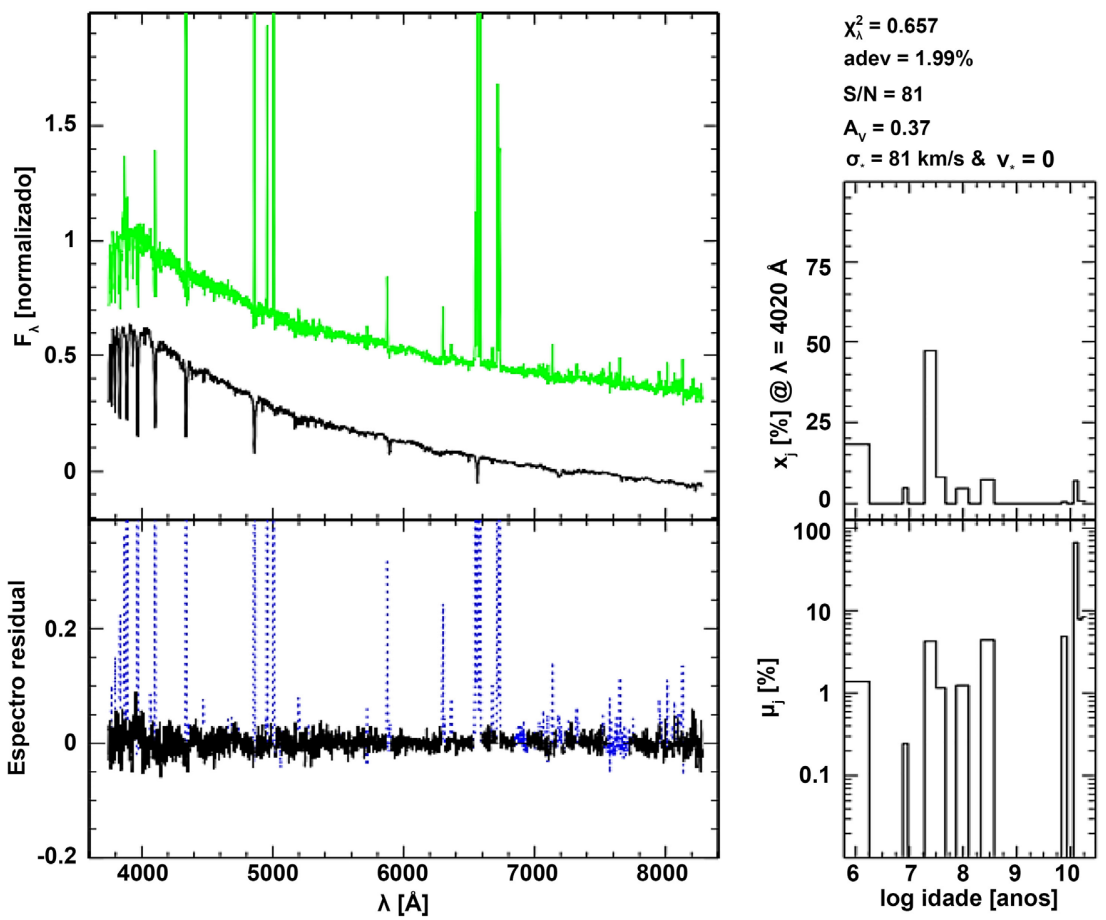

Figure 7. Like Figure 5, but for the late SDSS galaxy J110756.33+002305.54. 
We obtained a total of 80 spectra from 78 galaxies-the Mrk 1210 and NGC 7130 galaxies were each observed twice in different telescopes. Our sample consists of 43 Seyfert type 2 galaxies, Seyfert type 1 galaxies, and nine non-active galaxies shows in Figure 8. The spectra of the respective galaxies are shown in Figures 2-6. In addition to the sample galaxies, we also obtained spectra of stars to use as models for speed calibration. The stars were observed with the same instrumental configurations as the galaxies for all turns. This eliminates the need to make further corrections to the instrumental resolution for measuring velocity dispersion [15].

Data reduction was conducted by Dr. A. Garcia-Rissmann [8] and L. R. Vega [20]. Corrections were made for reading noise (bias), the pixel-by-pixel difference (flatfield), and dark current (dark) effects. As the standard procedure, the flow and wavelength calibration with the iraf tool package was applied. The fact

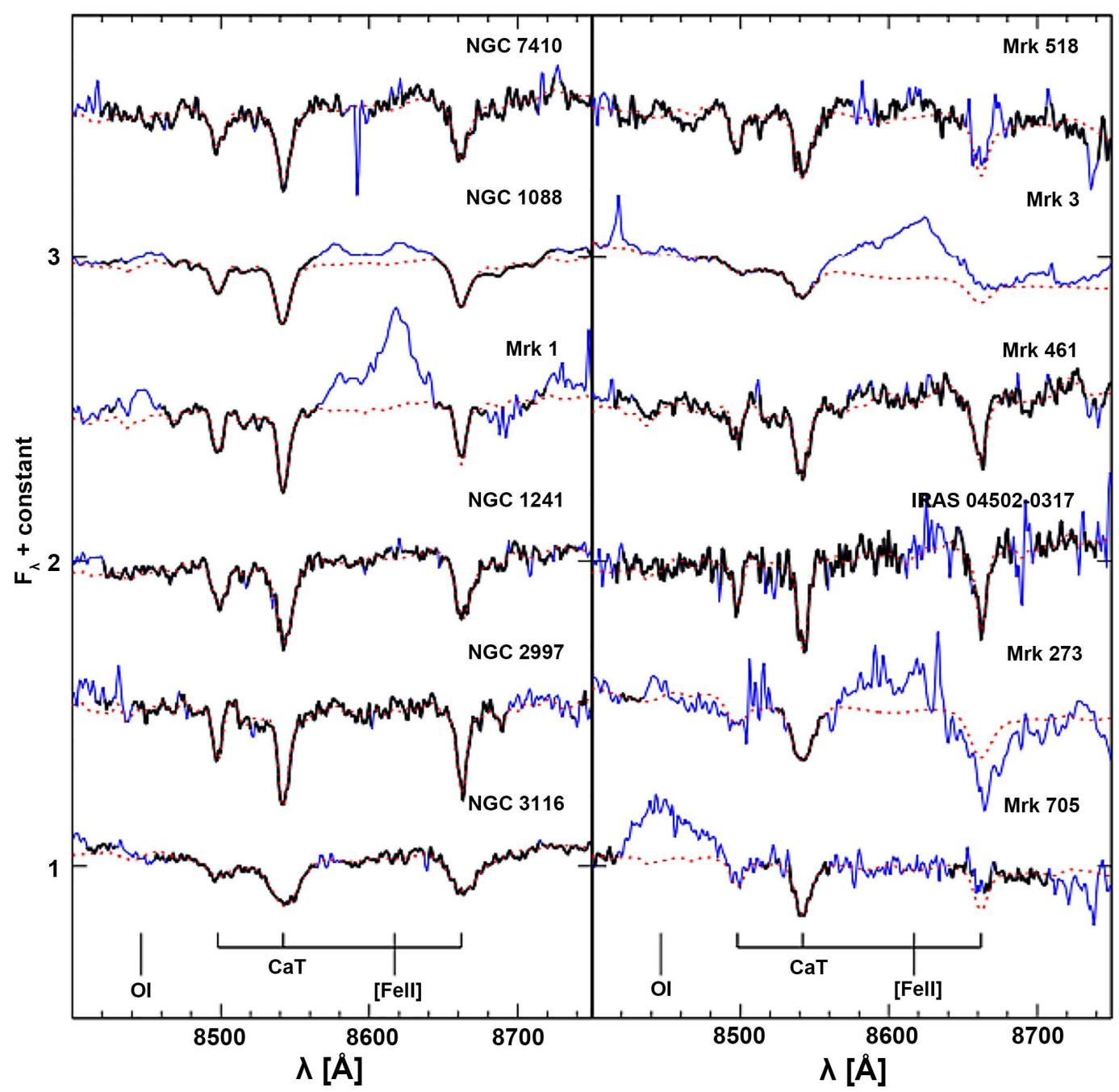

Figure 8. Examples of spectra modeled with a direct fit. In full-line, the observed spectra are graphed; in dotted line, the modeled spectra. The regions considered in the adjustments are drawn in a thicker line. The galaxies NGC 7410, NGC 1068, Mrk 1, NGC 1241, NGC 2997, and NGC 3115 are "a" quality; Mrk 516, Mrk 3 and Mrk 461, of "b" quality; and IRAS 04502-0317, Mrk 273, and Mrk 705, of "c" quality. 
that our spectra are in the near-infrared region caused us to have problems with fringing, that is, internal reflections in the CCD camera that create interference patterns in the image. In these cases, a careful procedure was adopted, following the recipe of Plait and Bohlin, to minimize the effect of the fringes. More details can be found in Garcia-Rissmann et al. [8]. To test to what extent fringes could interfere with our measurements of velocity dispersion $\sigma$, one of the main parameters of our analysis, we compared measurements in spectra with and without fringe correction. The difference between the $\sigma$ derivatives proved to be within the uncertainty estimates. All spectra were placed in the resting frame, using the mean redshift (redshift) measured in the Ca ii lines [31].

The Galactic reddening effect was also corrected for each galaxy, using NED data. All spectra presented in this chapter are nuclear. For all program objects, a three-pixel region centered on the luminosity peak of the galaxy was defined as nuclear. Defining the opening radius rab as the radius of a circle whose area comprises the area of our nuclear spectrum, we have, for our sample, rab $=50-700$ pc, and a median of $286 \mathrm{pc}$. Some spectra, after data reduction, present certain characteristics or artifacts that can hinder the analysis of the $\mathrm{Ca}$ ii region. Such characteristics are, for example, emission lines in the $\mathrm{Ca}$ ii region, excess noise, atmospheric spectrum remnants, and spurious effects [26].

For this reason, I, Dr. R. Cid Fernandes, and L. R. Vega classified the spectra, by visual inspection, according to the quality in the $\mathrm{Ca}$ ii region. "a" quality spectra are relatively clean and little affected by the aforementioned effects. Those of "b" quality are those in which one of the lines of $\mathrm{Ca}$ ii is contaminated. The quality " $c$ " is the most problematic, with at least two lines heavily compromised. The " $\mathrm{d}$ " spectra are those that have complexities beyond the analysis capacity of our study. Examples of the latter are the narrow-line Seyfert 1 galaxies with wide Ca ii lines in emission.

\section{Direct-Fit Method}

A tool increasingly used [33] to measure velocity dispersion is the direct fitting method (dfm, the acronym in English for the direct fitting method). This method consists of modeling the observed spectrum using a linear combination of elements of a base of standard velocity stars, whose lines of $\mathrm{Ca}$ ii are enlarged and displaced by the convolution with a Gaussian filter, according to the same formula used in the synthesis of stellar populations: with the following particularities: $\bullet M_{\lambda}$ is the synthetic spectrum. $\bullet M_{\lambda 0}$ is a normalization factor, defined as the total flux of the synthetic spectrum at wavelength $\lambda_{0}=8564 \AA . \cdot T_{j, \lambda}$ is the spectrum of the $t$ th $(j=1, \cdots, N)$ base component, normalized to $\lambda_{0}$. Each galaxy was modeled with a base that included only the observed velocity standard stars with the same instrumental configuration, so we do not need to make corrections for the resolution. Instrumental dog.

$$
M_{\lambda}=M_{\lambda 0}\left[\sum_{j=1}^{N} x_{j} T_{j} r_{\lambda}\right] \otimes G\left(v_{*}, \sigma_{*}\right)
$$


We also include taking into account the effects of stellar populations not included in the base, a continuum $C_{\lambda}$, which is a combination of power laws of the type $\lambda \beta . \bullet x_{j}$ is the fraction that each element $T_{j, \lambda}$ of the base contributes to the flow $M_{\lambda} \cdot r_{\lambda} \equiv 10-0.4\left(A_{\lambda}-A_{\lambda 0}\right)$ takes into account the effects of extinction by dust. In the case of the Ca ii triplet analysis, the measured value of $r_{\lambda}$ should not be taken into account, as we are working with a very narrow spectral range in which the effects cannot be measured, of dust. $-G(v, \sigma)$ is a Gaussian distribution of line-of-sight velocities, centered on $v$ and enlarged by $\sigma . \bullet \otimes$ expresses a convolution. The best fit is defined as the one that minimizes the $\chi$ two between the observed spectrum $\left(O_{\lambda}\right)$ and the model, as in Equation above.

Simply by defining the inverse of the noise as $w_{\lambda}=0$, individual masks are built for each galaxy in order to avoid spectral features that interfere with the adjustment, such as emission lines, bad pixels, and remnants of heaven. Furthermore, due to the construction of the algorithm's output files, the visualization and comparison between the observed spectrum and the obtained model are quite straightforward [17]. Figure 8 shows a depiction of this.

\section{The Effect of Masks}

Depending on the quality of the spectrum and the contamination of the $\mathrm{Ca}$ ii lines, the mask used in the direct fit method can have a considerable influence on the dispersion measurement. To speeds. In the preliminary tests with the masks, we were guided by the Work of Barth et al. (2002). In principle, we use a window from 8480 to $8690 \AA$, which completely includes the absorption lines of Ca ii. The region from 8560 to $8640 \AA$ was excluded from the analysis as it resulted in a poor fit. We test this mask for all our objects. After a visual inspection, we created another half-dozen "general masks", that is, masks used by all galaxies indiscriminately. After this first screening, we started to look more carefully at the galaxy by the galaxy. For each of the spectra, we verified the suitability of the "gm" and "gm5" masks.

We then built individual masks for each of the 80 spectra, excluding parts with low signal-to-noise, emission lines close to the $\mathrm{Ca}$ ii lines, or other effects that could interfere with the adjustment. Of the lines of $\mathrm{Ca}$ ii. For higher quality spectra (tipoa' type), it was noticed that the inclusion of continuum and other well-behaved regions around the lines of $\mathrm{Ca}$ ii do not interfere much in the measurement of $\sigma$. However, for some espectrob' and "c" type spectra, the fit was more coherent when concentrated in the lines of Ca ii. Anyway, the choice of the mask by visual inspection can be quite subjective. For this reason, I made three different individual masks for each galaxy, following slightly different criteria for each mask. The criteria differ mainly on whether or not to include continuous or noisier line wings. The variations in $\sigma$ according to the chosen individual mask, were of the order of $8 \mathrm{~km} / \mathrm{s}$, that is, within the estimated uncertainties $\Delta \sigma$. For "a" quality spectra, the variations are of the order of $5 \mathrm{~km} / \mathrm{s}$; for those of "b" quality, $9 \mathrm{~km} / \mathrm{s}$; for type "c," $14 \mathrm{~km} / \mathrm{s}$. 


\section{Direct-Fit Method}

The same comparative test between individual masks and general masks was not so promising, on the other hand. Excluding two complex cases, the variations in $\sigma$ due to the differences between general and individual masks were of the order of $17 \mathrm{~km} / \mathrm{s}$, still comparable to the uncertainties $\Delta \sigma$. For "a" quality spectra, the variations are of the order of $9 \mathrm{~km} / \mathrm{s}$; for those of " $b$ " quality, $30 \mathrm{~km} / \mathrm{s}$; for type "c," $27 \mathrm{~km} / \mathrm{s}$. The confection of individual masks, therefore, brings notable benefits for the reliability of measurements of $\sigma$, despite a certain amount of subjectivity involved. On the other hand, general masks, made according to the characteristics of the sample as a whole, are a good approximation for preliminary studies or those that do not need a very high degree of reliability. For large samples, where it is not possible to visually inspect all spectra, general masks can be used, taking certain precautions [12].

\section{Calculation of Uncertainties}

In this section, I detail the procedure for estimating uncertainties in $\sigma$ derived from the direct fit method. Although at first glance, this seems like an already well-defined subject in the literature, there are several details that can be complex and call into question the validity of a measure of $\Delta \sigma$ [21]. In addition to the work presented in this section, I have verified, throughout the study, whether other sources of uncertainty are consistent with $\Delta \sigma$ thus derived-as, for example, quantifying the effect of the choice of masks. I hope, in this way, to have exhaustively covered the main sources of doubt regarding $\Delta \sigma$. Unlike the crosscorrelation method and the direct-fit method used by, for example, Barth et al., our starlight direct fit method includes in its base all the stars available in an observation turn. This means that we already take into account, in the adjustment itself, the so-called template mismatch or the intrinsic difference between the stellar spectra that we use as velocity patterns. Thus, it is not sensible to have a measure of uncertainties only by analyzing different results for several stars, a procedure widely used in the literature [16].

Besides the way the algorithm was built, another problem in quantifying the uncertainty by the template mismatch is the choice of an adequate base of stars. If we are modeling the lines of $\mathrm{Ca}$ ii and include in the base very different stars, for example, hot stars of type $\mathrm{O}$ or even white dwarfs, what results could we expect for the dispersion of velocities with these adjustments? Obviously, it is not plausible to fit the absorption lines of the $\mathrm{Ca}$ ii triplet with spectra that do not have these lines. The uncertainty measured by the template mismatch, in this case, would be very high. On the other hand, if we use practically identical stars KOIII at the base, the measures of $\sigma$ will be very close, and the measurement uncertainty will be small. In this case, she's probably being underestimated. In other words, the choice of the set of comparison stars has a direct influence on the uncertainty estimate. Is this a criticism we make of the standard method of estimating $\Delta \sigma$ by template mismatch? With these two factors in mind, two sub-samples 
were then selected to verify the effect of template mismatch on direct fit. The first sub-sample comes from one of the shifts observed in the $1.52 \mathrm{~m}$ telescope of the ESO-La Silla. The base observed in this turn consists of stars of the types K0III, K3III, and K5III. The second sub-sample is one of the shifts observed in the $2.1 \mathrm{~m}$ KPNO telescope. The base of stars used is more diversified: they are of the types K0III, K3III, K5III, F0III, and G0III. For these sub-samples, we apply direct fit with bases consisting of only one star at a time, in addition to the power laws [13].

After obtaining the fit with the individual stars, we take the mean and the mean square deviation of $\sigma$ for each of the sub-samples. Figure 2 compares the results of $\sigma$ obtained by this procedure with the results measured with the base that includes all the stars of the turn. We use exactly the same individual masks for adjustments with different bases. For the ESO sub-sample, the uncertainty $\Delta \sigma \mathrm{tm}$ ? Due to the template mismatch, it is of the order of $5 \mathrm{~km} / \mathrm{s}$ or $30 \%$ larger than the measure adopted in this work $\Delta \sigma$, measured from the $\chi$ two distribution function, described below in this section. For the KPNO subsample, the mean square deviation of $\sigma$ due to the template mismatch is on the order of $14 \mathrm{~km} / \mathrm{s}$ or $100 \%$ larger than the adopted measure. Therefore, for the ESO sub-sample, with a less diversified starbase, the uncertainties derived from the template mismatch and the $\chi$ two curve method do not present significant statistical differences.

For the KPNO sub-sample, on the other hand, the difference in the measure of uncertainties starts to have some relevance. There are indications, as I had qualitatively argued earlier, that a very diversified base causes template mismatch measurements to result in overestimated uncertainties. Anyway, according to Barth et al., the uncertainties $\Delta \sigma \mathrm{tm}$ and $\Delta \sigma$ must be summed in quadrature. In this case, the measure of uncertainty presented in this work would be, at most, $40 \%$ higher. As described before, however, the direct-fit method used in this study already takes into account all available template stars and is, therefore, different from the method used by Barth et al. [33] does not seem to make much sense, in our case, to add $\Delta \sigma \mathrm{tm}$ to the estimated uncertainties.

In the KPNO sub-sample, there are type F stars, which clearly show the Paschen series. For these stars, the lines of Paschen Pa13, at $8665 \AA$, Pa15, $8545 \AA$, and Pa16, $8502 \AA$, are superimposed on the lines of the triplet of $\mathrm{Ca}$ ii, and make the measure of $\sigma$ of $\mathrm{Ca}$ ii is underestimated. It's not hard to understand why: if the fit algorithm tries to model a $\mathrm{Ca}$ ii line with a combined $\mathrm{Ca}$ ii and Paschen line, it will need to extend this by a smaller amount. Therefore, it is these underestimated measures, using individual stars, that cause the offset in Figure 2. When the modeling is done with a base of stars K, F, and G, on the other hand, the program gives preference to those that do not have Paschen lines and, therefore, estimates a correct value for $\sigma$ of Ca ii.

The question is, therefore, how to quantify the uncertainties from the direct fits with the complete starbase. According to Press et al., one way to estimate the error bar is from the $\chi$ two distribution function. Because we are interested in uncer- 
tainty in just one parameter, $\sigma$, this method provides a simple and straightforward procedure. In this case, a variation of $\Delta \chi^{2}=1$ is statistically equivalent to an extension that covers $68.3 \%$ of a data set in a normal distribution. The method is computationally expensive but not too complicated. For each galaxy in our sample, we need to obtain a curve of $\chi$ two as a function of $\sigma$. We start from the $\sigma$, the best value obtained with the model that minimizes $\chi^{2}$. Let's call this $\chi$ two $\chi^{2}$ min. We travel an interval of $40 \mathrm{~km} / \mathrm{s}$ around the $\sigma$, best. In this window, we set the value of $\sigma$, adjust the other parameters of the model and recalculate $\chi^{2}$ [33]. Thus, we have a distribution of $\chi$ two as a function of the parameter $\sigma$, as shown in Figure 9 and Figure 10.

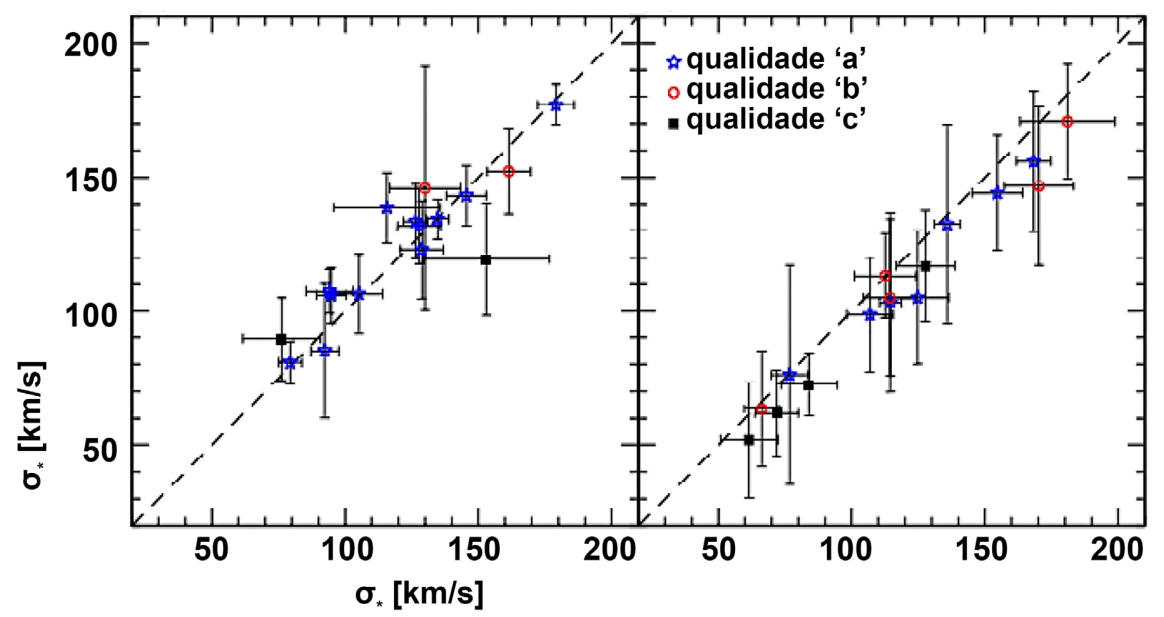

Figure 9. Template mismatch results for ESO and KPNO $2.1 \mathrm{~m}$ sub-samples.

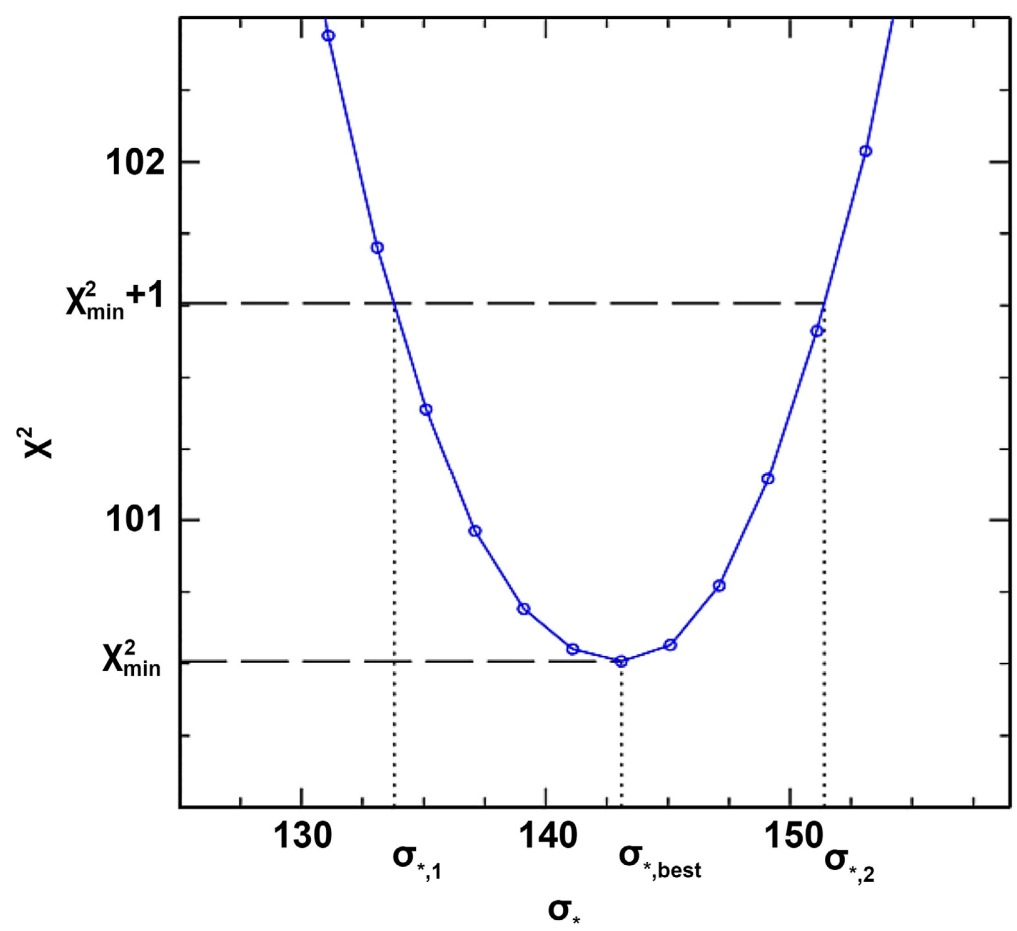

Figure 10. Typical curve of $\chi$ two as a function of $\sigma$. 


\section{Discussion and Conclusion}

The discovery of an empirical relationship between the mass of the central black hole (M•) and $\sigma$ was the main guide of our detailed study of the calcium triplet region. Therefore, the search for more accurate methods for calculating the dispersion of velocities, in addition to the careful study of uncertainties. So one of the following steps is to calculate $\mathrm{M} \bullet$ for our objects and relate it to other properties. It has recently been questioned whether there are "intermediate" black holes, that is, not as massive as those usually found in the center of galaxies. We suspect that this possibility is worth investigating for at least one object (NGC 4748) in our sample. However, it should be remembered that our instrumental scattering is not very convenient to detect $\sigma$ small.

Another critical study to be done with the calcium triplet is calculating the mass/luminosity (M/L) ratio from $\sigma$ and photometric information. Thus, one can further investigate the stellar populations of the active galaxies. This possibility is exciting for type 1 Seyferts, for which traditional stellar population diagnoses are not feasible. For the study of sdss, we have as much to improve our preliminary results as to investigate another immense range of analyses. The mask detection program, for example, can receive an even more complete list of emission lines to be detected, including recombination lines important in the study of chemical abundances, like $\mathrm{C}$ and $\mathrm{O}$. Furthermore, the program can be improved to fit the lines more precisely by calculating other parameters of the Gaussian with which we try to model the lines-at the moment, we just adjust their amplitude. Also, we can use it for weak line detection [29].

As we've seen, galaxy mid-range spectra allow you to see certain spectral features that are hidden by noise in individual spectra-both adjustment problems and weak emission lines. That is, we can use the program that masks lines with slight modifications (or perhaps none at all) to detect these faint lines in medium spectra automatically. We also fit new models for our sample of $\sim 355,000$ objects, this time with a simple stellar population base that excludes populations with very low metallicity $(Z=1 / 200$ e $1 / 50 Z)$ included in the analysis presented here. These populations have important effects on some parameters, such as $M / L$, mass, and average age. However, their spectra in the Bruzual and Charlot [34] models are computed relatively coarsely due to the lack of a complete library of stellar spectra with such metallicities. Therefore, we found it convenient to investigate their effect on our adjustments. This same group will even soon release new versions of metal-poor populations.

As for the preliminary results presented here, there are several details that we have not yet included in our calculations for the BPT diagnostic diagram and star formation history. We need, for example, to further investigate the completeness of the sample and correct our timescale to a cosmological timescale. We hope, therefore, to obtain SFRs that show in more detail the evolution of galaxies in the local universe. Therefore, the immediately subsequent steps are to detail our studies of sdss more rigorously. Furthermore, as we have already men- 
tioned, there are many other synthesis products that we can investigate, and it would be impossible to list all these branches here.

\section{Conflicts of Interest}

The author declares no conflicts of interest regarding the publication of this paper.

\section{References}

[1] Adhikari, S., Dalal, N., More, S. and Wetzel, A. (2019) Kinematics of Cluster Galaxies and Their Relation to Galaxy Evolution. The Astrophysical Journal, 878, Article No. 9. https://doi.org/10.3847/1538-4357/ab1a39

[2] Bland-Hawthorn, J. and Gerhard, O. (2016) The Galaxy in Context: Structural, Kinematic, and Integrated Properties. Annual Review of Astronomy and Astrophysics, 54, 529-596. https://doi.org/10.1146/annurev-astro-081915-023441

[3] Cappellari, M. (2016) Structure and Kinematics of Early-Type Galaxies from Integral Field Spectroscopy. Annual Review of Astronomy and Astrophysics, 54, 597-665. https://doi.org/10.1146/annurev-astro-082214-122432

[4] Coccato, L., Jaffé, Y.L., Cortesi, A., Merrifield, M., Johnston, E., Rodriguez del Pino, B., et al. (2020) Formation of S0s in Extreme Environments I: Clues from Kinematics and Stellar Populations. Monthly Notices of the Royal Astronomical Society, 492, 2955-2972. https://doi.org/10.1093/mnras/stz3592

[5] Cortesi, A., Merrifield, M.R., Coccato, L., Arnaboldi, M., Gerhard, O., Bamford, S., et al. (2013) Planetary Nebula Spectrograph Survey of S0 Galaxy Kinematics-II. Clues to the Origins of S0 Galaxies. Monthly Notices of the Royal Astronomical Society, 432, 1010-1020. https://doi.org/10.1093/mnras/stt529

[6] Font, J., Beckman, J.E., Martínez-Valpuesta, I., Borlaff, A.S., James, P.A., Díaz-García, S., et al. (2017) Kinematic Clues to Bar Evolution for Galaxies in the Local Universe: Why the Fastest Rotating Bars Are Rotating Most Slowly. The Astrophysical Journal, 835, Article No. 279. https://doi.org/10.3847/1538-4357/835/2/279

[7] Gadotti, D.A., Bittner, A., Falcón-Barroso, J., Méndez-Abreu, J., Kim, T., Fragkoudi, F., et al. (2020) Kinematic Signatures of Nuclear Discs and Bar-Driven Secular Evolution in Nearby Galaxies of the MUSE TIMER Project. Astronomy \& Astrophysics, 643, Article No. A14. https://doi.org/10.1051/0004-6361/202038448

[8] Garcia-Rissmann, A., Vega, L.R., Asari, N.V., Fernandes, R.C., Schmitt, H., González Delgado, R.M. and Storchi-Bergmann, T. (2005) An Atlas of Calcium Triplet Spectra of Active Galaxies. Monthly Notices of the Royal Astronomical Society, 359, 765-780. https://doi.org/10.1111/j.1365-2966.2005.08957.x

[9] Hung, C.L., Hayward, C.C., Yuan, T., Boylan-Kolchin, M., Faucher-Giguère, C.A., Hopkins, P.F., et al. (2019) What Drives the Evolution of Gas Kinematics in StarForming Galaxies? Monthly Notices of the Royal Astronomical Society, 482, 51255137. https://doi.org/10.1093/mnras/sty2970

[10] Hung, C.L., Rich, J.A., Yuan, T., Larson, K.L., Casey, C.M., Smith, H.A., et al. (2015) Kinematic Classifications of Local Interacting Galaxies: Implications for the Merger/Disk Classifications at High-Z. The Astrophysical Journal, 803, Article No. 62. https://doi.org/10.1088/0004-637X/803/2/62

[11] Kassin, S.A., Brooks, A., Governato, F., Weiner, B.J. and Gardner, J.P. (2014) Kinematic Evolution of Simulated Star-Forming Galaxies. The Astrophysical Journal, 790, Article No. 89. https://doi.org/10.1088/0004-637X/790/2/89 
[12] Katkov, I.Y., Sil'chenko, O.K. and Afanasiev, V.L. (2014) Decoupled Gas Kinematics in Isolated S0 Galaxies. Monthly Notices of the Royal Astronomical Society, 438, 2798-2803. https://doi.org/10.1093/mnras/stt2365

[13] Kirby, E.N., Gilbert, K.M., Escala, I., Wojno, J., Guhathakurta, P., Majewski, S.R. and Beaton, R.L. (2020) Elemental Abundances in M31: The Kinematics and Chemical Evolution of Dwarf Spheroidal Satellite Galaxies. The Astronomical Journal, 159, Article No. 46. https://doi.org/10.3847/1538-3881/ab5f0f

[14] Lapi, A., Pantoni, L., Zanisi, L., Shi, J., Mancuso, C., Massardi, M., et al. (2018) The Dramatic Size and Kinematic Evolution of Massive Early-Type Galaxies. The Astrophysical Journal, 857, Article No. 22. https://doi.org/10.3847/1538-4357/aab6af

[15] MacKay, D.J.C. (2003) Information Theory, Inference, and Learning Algorithms. Cambridge University Press, Cambridge.

[16] Nelson, C.H., Green, R.F., Bower, G., Gebhardt, K. and Weistrop, D. (2004) The Relationship Between Black Hole Mass and Velocity Dispersion in Seyfert 1 Galaxies. The Astrophysical Journal, 615, Article No. 652. https://doi.org/10.1086/424657

[17] Schulze, F., Remus, R.S., Dolag, K., Bellstedt, S., Burkert, A. and Forbes, D.A. (2020) Kinematics of Simulated Galaxies II: Probing the Stellar Kinematics of Galaxies out to Large Radii. Monthly Notices of the Royal Astronomical Society, 493, 3778-3799. https://doi.org/10.1093/mnras/staa511

[18] Tissera, P.B., Scannapieco, C., Beers, T.C. and Carollo, D. (2013) Stellar Haloes of Simulated Milky-Way-Like Galaxies: Chemical and Kinematic Properties. Monthly Notices of the Royal Astronomical Society, 432, 391-3400. https://doi.org/10.1093/mnras/stt691

[19] Toloba, E., Boselli, A., Peletier, R.F., Falcón-Barroso, J., van de Ven, G. and Gorgas, J. (2012) Formation and Evolution of Dwarf Early-Type Galaxies in the Virgo Cluster-II. Kinematic Scaling Relations. Astronomy \& Astrophysics, 548, Article No. A78. https://doi.org/10.1051/0004-6361/201218944

[20] Vega, O., Bressan, A., Panuzzo, P., Rampazzo, R., Clemens, M., Granato, G.L., et al. (2010) Unusual PAH Emission in Nearby Early-Type Galaxies: A Signature of an Intermediate-Age Stellar Population? The Astrophysical Journal, 721, 1090-1104. https://doi.org/10.1088/0004-637X/721/2/1090

[21] Webb, J.J. and Vesperini, E. (2018) The Structural and Kinematic Evolution of Central Star Clusters in Dwarf Galaxies and Their Dependence on Dark Matter Halo Profiles. Monthly Notices of the Royal Astronomical Society, 479, 3708-3714. https://doi.org/10.1093/mnras/sty1723

[22] Wisnioski, E., Schreiber, N.F., Wuyts, S., Wuyts, E., Bandara, K., Wilman, D., et al. (2015) The Kmos3d Survey: Design, First Results, and the Evolution of Galaxy Kinematics from $0.7 \leq \mathrm{z} \leq 2.7$. The Astrophysical Journal, 799, Article No. 209.

https://doi.org/10.1088/0004-637X/799/2/209

[23] Kauffmann, G., Heckman, T.M., White, S.D., Charlot, S., Tremonti, C., Brinchmann, J., et al. (2003) Stellar Masses and Star Formation Histories for 105 Galaxies from the Sloan Digital Sky Survey. Monthly Notices of the Royal Astronomical Society, 341, 33-53. https://doi.org/10.1046/j.1365-8711.2003.06291.x

[24] Kauffmann, G., Heckman, T.M., Tremonti, C., Brinchmann, J., Charlot, S., White, S.D., et al. (2003) The Host Galaxies of Active Galactic Nuclei. Monthly Notices of the Royal Astronomical Society, 346, 1055-1077. https://doi.org/10.1111/j.1365-2966.2003.07154.x

[25] Kauffmann, G., Heckman, T.M., White, S.D., Charlot, S., Tremonti, C., Peng, E.W., et al. (2003) The Dependence of Star Formation History and Internal Structure on 
Stellar Mass for 105 Low-Redshift Galaxies. Monthly Notices of the Royal Astronomical Society, 341, 54-69. https://doi.org/10.1046/j.1365-8711.2003.06292.x

[26] Shen, S., Mo, H.J., White, S.D., Blanton, M.R., Kauffmann, G., Voges, W., et al. (2003) The Size Distribution of Galaxies in the Sloan Digital Sky Survey. Monthly Notices of the Royal Astronomical Society, 343, 978-994. https://doi.org/10.1046/j.1365-8711.2003.06740.x

[27] Heavens, A., Panter, B., Jimenez, R. and Dunlop, J. (2004) The Star-Formation History of the Universe from the Stellar Populations of Nearby Galaxies. Nature, 428, 625-627. https://doi.org/10.1038/nature02474

[28] Panter, B., Heavens, A.F. and Jimenez, R. (2003) Star Formation and Metallicity History of the SDSS Galaxy Survey: Unlocking the Fossil Record. Monthly Notices of the Royal Astronomical Society, 343, 1145-1154. https://doi.org/10.1046/j.1365-8711.2003.06722.x

[29] Cid Fernandes, R., González Delgado, R.M., Storchi-Bergmann, T., Martins, L.P. and Schmitt, H. (2005) The Stellar Populations of Low-Luminosity Active Galactic Nuclei-III. Spatially Resolved Spectral Properties. Monthly Notices of the Royal Astronomical Society, 356, 270-294. https://doi.org/10.1111/j.1365-2966.2004.08452.x

[30] Fusaro, A.F., Matthew, L., Smith, N.A., Curtin, S.J., Dedic-Hagan, J., Ellacott, G.A., et al. (2006) RNA Interference-Inducing Hairpin RNAs in Plants Act through the Viral Defence Pathway. EMBO Reports, 7, 1168-1175. https://doi.org/10.1038/sj.embor.7400837

[31] Stasińska, G., Fernandes, R.C., Mateus, A., Sodré, L. and Asari, N.V. (2006) Semi-Empirical Analysis of Sloan 840 Digital Sky Survey Galaxies-III. How to Distinguish AGN Hosts. Monthly Notices of the Royal Astronomical Society, 371, 972-982. https://doi.org/10.1111/j.1365-2966.2006.10732.x

[32] Gomes, R., Levison, H.F., Tsiganis, K. and Morbidelli, A. (2005) Origin of the Cataclysmic Late Heavy Bombardment Period of the Terrestrial Planets. Nature, 435, 466-469. https://doi.org/10.1038/nature03676

[33] Barth, A.J., Ho, L.C. and Sargent, W.L. (2002) Stellar Velocity Dispersion and Black Hole Mass in the Blazar Markarian 501. The Astrophysical Journal Letters, 566, Article No. L13. https://doi.org/10.1086/339452

[34] Bruzual, G. and Charlot, S. (2003) Stellar Population Synthesis at the Resolution of 2003. Monthly Notices of the Royal Astronomical Society, 344, 1000-1028. https://doi.org/10.1046/j.1365-8711.2003.06897.x 